Pacific Journal of Mathematics

ON THE DISTRIBUTION OF WEIERSTRASS POINTS ON 


\title{
ON THE DISTRIBUTION OF WEIERSTRASS POINTS ON IRREDUCIBLE RATIONAL NODAL CURVES
}

\author{
John B. Little AND Kathryn A. Furio
}

Let $X$ be an irreducible rational nodal curve of arithmetic genus $g \geq 2$, and let $\mathscr{L}$ be a non-special, effective invertible sheaf on $X$. Let $W(\mathscr{L})$ denote the set of smooth Weierstrass points of $\mathscr{L}$ and all its positive tensor powers on $X$. In this paper, we study the distribution of $W(\mathscr{L})$ on $X$. In particular, we will show that $W(\mathscr{L})$ is not dense on $X$, generalizing an example of R. F. Lax.

1. Introduction. In a recent series of papers ([2], [3], [4]), R. F. Lax and $\mathrm{C}$. Widland have defined Weierstrass points for invertible sheaves on integral, projective Gorenstein curves over $\mathbf{C}$. They use a method generalizing the classical definition of the Weierstrass points of the canonical sheaf on a smooth curve via Wronskians. In particular, they show that if $X$ is an integral, projective Gorenstein curve, and $\mathscr{L}$ is an invertible sheaf on $X$, then a smooth point $P \in X$ is a Weierstrass point of $\mathscr{L}$ if and only if

$$
\operatorname{dim} H^{0}(X, \mathscr{L}(-s P))>0,
$$

where $s=\operatorname{dim} H^{0}(X, \mathscr{L})$. On the other hand, if $s \geq 2$, the singular points of $X$ are automatically Weierstrass points of $\mathscr{L}$ of high Weierstrass weight. (See Propositions 2 and 3 of [3].)

The goal of the present note is to prove a general result about the distribution of the smooth Weierstrass points of an invertible sheaf $\mathscr{L}$ and all its positive tensor powers in the case that $X$ is an irreducible rational nodal curve. This particular question was suggested by an example in [3], in which it is shown that for a particular $\mathscr{L}$ on a particular rational nodal curve of arithmetic genus 2 , the set

$W(\mathscr{L})=\left\{P \in X \mid X\right.$ is a smooth Weierstrass point of $\mathscr{L}^{\otimes n}$

for some $n \geq 1\}$

avoids a small disk in the normalization of $X$ (that is, $\left.\mathbb{P}^{1}\right)$. This situation is quite different from the case of smooth curves $X$, where B. Olsen ([6]) had previously shown that if $\operatorname{deg}(\mathscr{L})>0$, then the analogous set $W(\mathscr{L})$ is dense in the complex topology on $X$. 
We will show that if $X$ is any irreducible rational nodal curve of arithmetic genus $g \geq 2$, and $\mathscr{L}$ is any invertible sheaf on $X$ with $\operatorname{dim} H^{0}(X, \mathscr{L})>0$, and $\operatorname{dim} H^{1}(X, \mathscr{L})=0$, then $W(\mathscr{L})$ is never dense in $X$. In fact, we will show that the limit points of $W(\mathscr{L})$ always lie on a real one-dimensional subset of $X$. (On the normalization of $X-\mathbb{P}^{1}$ viewed as the Riemann sphere-this set is a union of circles). The proof uses an explicit form of the equation of the theta-divisor in the generalized Jacobian of $X$, given in Mumford's book [5], and a characterization of the smooth Weierstrass points of $\mathscr{L}$ borrowed from Olsen's work for smooth $X$.

In $\S 2$ we fix our notation and gather some preliminary results. The proof of our main result follows in $\S 3$.

2. Weierstrass points on rational nodal curves. Let $X$ be the irreducible rational nodal curve formed by identifying $g$ pairs of distinct points $\left\{b_{i}, c_{i}\right\}$ on $\mathbb{P}^{1}$ (the Riemann sphere, or extended complex plane). For notational simplicity, we assume that none of the points $b_{i}$ or $c_{i}$ is $\infty$.

Let $\omega$ denote the dualizing sheaf of $X$. Then $H^{0}(X, \omega)$ is spanned by the differentials $\omega_{j}=d z /\left(z-b_{j}\right)-d z /\left(z-c_{j}\right)(j=1, \ldots, g)$. Moreover, with this choice of basis, the period lattice $\Lambda$ of $X$ is generated by the $g$ vectors in $\{(2 \pi i, 0, \ldots, 0),(0,2 \pi i, 0, \ldots, 0), \ldots$, $(0, \ldots, 0,2 \pi i)\}$. Hence the generalized Jacobian $J(X)=\mathbf{C}^{g} / \Lambda$ may be identified with $\left(\mathbf{C}^{*}\right)^{g}$.

Let $X_{0}$ denote the set of smooth points of $X$, and let $x_{0}=\infty \in X_{0}$. As on smooth curves, we define an Abel mapping $\varphi: X_{0} \rightarrow J(X)$, using $x_{0}$ as basepoint, by

$$
\begin{aligned}
\varphi(x) & =\left(\exp \left(\int_{x_{0}}^{x} \omega_{1}\right), \ldots, \exp \left(\int_{x_{0}}^{x} \omega_{g}\right)\right) \\
& =\left(\left(x-b_{1}\right) /\left(x-c_{1}\right), \ldots,\left(x-b_{g}\right) /\left(x-c_{g}\right)\right) .
\end{aligned}
$$

We may extend $\varphi$ to a mapping on effective divisors with support in $X_{0}$ using the group operation in $J(X)$ :

$$
\begin{aligned}
\varphi: X_{0}^{(m)} & \rightarrow J(X), \\
\sum n_{k} x_{k} & \rightarrow\left(\prod_{k}\left[\left(x_{k}-b_{1}\right) /\left(x_{k}-c_{1}\right)\right]^{n_{k}}, \ldots,\right. \\
& \left.\prod_{k}\left[\left(x_{k}-b_{g}\right) /\left(x_{k}-c_{g}\right)\right]^{n_{k}}\right) .
\end{aligned}
$$


Finally, if $D=D_{1}-D_{2}$ with $D_{i}$ effective we define

$$
\varphi(D)=\varphi\left(D_{1}\right)\left(\varphi\left(D_{2}\right)\right)^{-1} \text {. }
$$

The image of $X_{0}^{(g-1)}$ under $\varphi$, or its Zariski closure in a compactification of $J(X)$, is the analog of the theta divisor in the Jacobian of a smooth curve, and as Mumford shows in [5], we can obtain an analog of the theta function, whose zero locus $\Theta \subset J(X)$ contains $\varphi\left(X_{0}^{(g-1)}\right)$.

LeMMA 1 ([5], pp. 250-251). Let $\lambda_{i}=e^{z_{i}}(i=1, \ldots, g)$ be coordinates on $J(X)$, and let

$$
\tau_{X}\left(\lambda_{1}, \ldots, \lambda_{g}\right)=\operatorname{det}\left[\begin{array}{ccc}
1-\lambda_{1} & \cdots & 1-\lambda_{g} \\
b_{1}-c_{1} \lambda_{1} & \cdots & b_{g}-c_{g} \lambda_{g} \\
& \vdots & \\
b_{1}^{g-1}-c_{1}^{g-1} \lambda_{1} & \cdots & b_{g}^{g-1}-c_{g}^{g-1} \lambda_{g}
\end{array}\right] .
$$

Then $\tau_{X}\left(\lambda_{1}, \ldots, \lambda_{g}\right)=0$ defines an irreducible divisor $\Theta \subset J(X)$ that contains $\varphi\left(X_{0}^{(g-1)}\right)$.

We will use the theta function $\tau_{X}$ to obtain a criterion for smooth Weierstrass points as follows. This is analogous to a result used by Olsen ([6], p. 362), and generalizes Lemma 2 of [3].

LEMMA 2. Let $\mathscr{L}$ be an invertible sheaf on $X$ such that $\operatorname{dim} H^{1}(X, \mathscr{L})=0$, and $s=\operatorname{dim} H^{0}(X, \mathscr{L})>0$. If $P \in X_{0}$ is a smooth Weierstrass point of $\mathscr{L}$, then $\varphi(\mathscr{L})(\varphi(P))^{-s} \in \Theta$.

Proof. We begin with a comment regarding the notation $\varphi(\mathscr{L})$ in the statement of the lemma. The generalized Jacobian $J(X)$ is isomorphic to $\mathrm{Pic}^{0}(X)$, the group of isomorphism classes of invertible sheaves of degree 0 on $X$. Given any invertible sheaf $\mathscr{L}$ on $X$, $\mathscr{L} \cong \mathscr{O}_{X}(D)$ for some divisor $D$ supported in $X_{0}$. Moreover, if $D_{1}$ and $D_{2}$ are linearly equivalent divisors supported in $X_{0}$, then the Abel Theorem for $X$ implies that $\varphi\left(D_{1}\right)=\varphi\left(D_{2}\right)$. Thus, we may define $\varphi(\mathscr{L})$ to be the image $\varphi(D)$ for any $D$ supported on $X_{0}$ with $\mathscr{L} \cong \mathscr{O}_{X}(D)$. Similarly, we can define $\varphi(D)$ for any Cartier divisor $D$ on $X$.

Now, we consider the situation described in the hypotheses of the lemma. Since $P$ is a smooth Weierstrass point of $\mathscr{L}$, there exists a nonzero section $\sigma \in H^{0}(X, \mathscr{L}(-s P))$. Then $E=\operatorname{div}(\sigma)-s P$ is an effective Cartier divisor on $X$. Its degree is $\operatorname{deg}(\mathscr{L})=s=g-1$ by the 
Riemann-Roch Theorem for Gorenstein curves (using the assumption that $\mathscr{L}$ is non-special).

Even though $E$ may not be supported entirely in $X_{0}$, since it is a Cartier divisor, $E$ can be viewed as a limit of effective divisors of degree $g-1$ supported in $X_{0}$, as in [1]. The image $\varphi(E)$ can then be computed by a limiting process as follows. By the linearity of the abelian sums, we can reduce to the case in which $Q$ is a node of $X, E$ is supported at $Q$, and $f \in \mathscr{O}_{X, Q}$ is a local equation for $E$. For $\varepsilon \in \mathbf{C} \backslash\{0\}$ sufficiently small in absolute value, the divisor $E_{\varepsilon}=\operatorname{div}(f-\varepsilon)$ will consist of $\operatorname{deg}(E)$ smooth points. As in Theorem 2 of [1], we will have $\varphi(E)=\lim _{\varepsilon \rightarrow 0} \varphi\left(E_{\varepsilon}\right)$.

Thus $\varphi(\mathscr{L})(\varphi(P))^{-s}=\varphi(E)$ is in the closure of $\varphi\left(X_{0}^{(g-1)}\right)$ in $J(X)$, and the lemma is proved.

We will use the following notation. When we expand the determinant for $\tau_{X}$, we have:

$$
\tau_{X}\left(\lambda_{1}, \ldots, \lambda_{g}\right)=\sum_{I \subseteq\{1, \ldots, g\}} a_{I} \cdot \prod_{i \in I} \lambda_{i},
$$

for some $a_{I} \neq 0$ in $\mathbf{C}$. (See [5], page 252. $a_{\{1, \ldots, g\}}$ is the Vandermonde determinant defined by the $c_{j}, a_{\varnothing}$ is the Vandermonde determinant of the $b_{j}$, and the other coefficients are various "mixed" Vandermonde determinants in the $c_{j}$ and $b_{j}$.)

Say $\varphi(\mathscr{L})=\left(d_{1}, \ldots, d_{g}\right) \in J(X)$. Then we have the following corollary.

Corollary 3. Let $\mathscr{L}$ be as in Lemma 2. If $P$ is a smooth Weierstrass point of order $n$ of $\mathscr{L}$ (that is, a Weierstrass point of the invertible sheaf $\left.\mathscr{L}^{\otimes n}\right)$, and $s(n)=\operatorname{dim} H^{0}\left(X, \mathscr{L}^{\otimes n}\right)$, then

$$
0=\sum_{I \subseteq\{1, \ldots, g\}} a_{I} \cdot \prod_{i \in I} d_{i}^{n} v_{i}^{s(n)}
$$

where $v_{i}$ is the reciprocal of the ith component of $\varphi(x)$, that is,

$$
v_{i}=\left(x-c_{i}\right) /\left(x-b_{i}\right) .
$$

3. The limit points of $W(\mathscr{L})$. We let $W(\mathscr{L})$ denote the set of all smooth Weierstrass points of $\mathscr{L}^{\otimes n}$, for all $n \geq 1$, as in the introduction. By Lemma $3, W(\mathscr{L})$ is the set of roots of all the equations $(*)$ 
for all $n \geq 1$. By the Riemann-Roch Theorem, we have

$$
s(n)=\operatorname{dim} H^{0}(X, \mathscr{L})=n \cdot \operatorname{deg}(\mathscr{L})+1-g .
$$

In this section, we will prove the following result about the distribution of the limit points of $W(\mathscr{L})$ on $X$.

THEOREM 4. Let $\rho_{j}=\left|d_{j}\right|^{-1 / \operatorname{deg}(\mathscr{L})}$ for each $j=1, \ldots, g$. Then the limit points of $W(\mathscr{L})$ all lie on the locus $L=\left\{x|| v_{j}(x) \mid=\rho_{j}\right.$ for some $j\}$. (Via the normalization map, on the Riemann sphere, this locus consists of (at most) g circles.)

Proof. Let $x \in \mathbb{P}^{1}$ be any point lying outside the locus $L$. We claim that for all $n$ sufficiently large (the bound depending on the location of $x), x$ is not a Weierstrass point of order $n$ of $\mathscr{L}$. We will prove this by showing that for all $n$ sufficiently large, $x$ cannot be a root of (*).

Consider first the case where $\left|v_{j}(x)\right|>\rho_{j}$ for all $j$. Then for all $n,\left|d_{j}\right|^{n}\left|v_{j}\right|^{n \cdot \operatorname{deg}(\mathscr{L})}>1$. Hence by taking $n$ large,

$$
\left|d_{j}^{n} v_{j}^{s(n)}\right|=\left|d_{j}\right|^{n}\left|v_{j}\right|^{n \cdot \operatorname{deg}(\mathscr{L})+1-g}
$$

can be made arbitrarily large. Thus, in the expression on the righthand side in equation $(*)$, the term $a_{\{1, \ldots, g\}} \cdot \prod_{i=1}^{g} d_{i}^{n} v_{i}^{s(n)}$ is the one that grows the fastest with $n$, and for $n$ large enough, we can make its absolute value larger than the sum of the absolute values of the other terms. By the triangle inequality, $x$ cannot be a root of $(*)$ if $n$ is chosen to be large enough.

Now, if $\left|v_{j}(x)\right|<\rho_{j}$ for some $j$, we use the same argument, but the fastest growing term will be the one for which $I=\left\{j|| v_{j}(x) \mid>\rho_{j}\right\}$. In all cases, for all $n$ sufficiently large, we see that $x$ cannot be a root of $(*)$.

Thus the limit points of $W(\mathscr{L})$ must all lie on the locus $L \subset \mathbb{P}^{1} . \square$

As a result, $W(\mathscr{L})$ is never dense in $X$. Some numerical experiments we have performed seem to indicate that the set of limit points of $W(\mathscr{L})$ may have a very complicated structure, but we are unable to say more at this time.

The first author has recently proved an analogous result for cuspidal rational curves as well, which will appear elsewhere. In that case too, the set $W(\mathscr{L})$ fails to be dense. 


\section{REFERENCES}

[1] V. Ancona, On the Abel-Jacobi Theorem for Singular Curves (Italian), Seminari di Geometria 1984, Univ. Stud. Bologna, Bologna, (1985), 3-6.

[2] R. F. Lax, Weierstrass points on rational nodal curves, Glasgow Math. J., 29 (1987), 131-140.

[3] - On the distribution of Weierstrass points on singular curves, Israel J. Math., 57 (1987), 107-115.

[4] R. F. Lax and C. Widland, Weierstrass points on rational nodal curves of arithmetic genus 3, Canad. Math. Bull., 30 (1987), 286-294.

[5] D. Mumford, Tata Lectures on Theta II, Birkhauser, Boston, 1983.

[6] B. Olsen, On Higher Order Weierstrass Points, Ann. of Math., 95 (1972), 357364.

Received September 9, 1988.

College of the Holy Cross

WORCESTER, MA 01610 


\section{PACIFIC JOURNAL OF MATHEMATICS EDITORS}

\author{
V. S. VARADARAJAN \\ (Managing Editor) \\ University of California \\ Los Angeles, CA 90024-1555-05 \\ Herbert Clemens \\ University of Utah \\ Salt Lake City, UT 84112 \\ Thomas ENRIGHT \\ University of California, San Diego \\ La Jolla, CA 92093
}

R. FINN

Stanford University

Stanford, CA 94305

Hermann FlaschKa

University of Arizona

Tucson, AZ 85721

VAUGHAN F. R. Jones

University of California

Berkeley, CA 94720

STEVEN KERCKHOFF

Stanford University

Stanford, CA 94305
C. C. MOORE

University of California

Berkeley, CA 94720

Martin SCharlemanN

University of California

Santa Barbara, CA 93106

HAROLd STARK

University of California, San Diego

La Jolla, CA 92093

\section{ASSOCIATE EDITORS}
R. ARENS
E. F. BECKENBACH
B. H. NeumanN
F. WoLF
(1904-1989)
K. YoshidA (1906-1982)

\section{SUP}

UNIVERSITY OF ARIZONA

UNIVERSITY OF BRITISH COLUMBIA

CALIFORNIA INSTITUTE OF TECHNOLOGY

UNIVERSITY OF CALIFORNIA

MONTANA STATE UNIVERSITY

UNIVERSITY OF NEVADA, RENO

NEW MEXICO STATE UNIVERSITY

OREGON STATE UNIVERSITY
UNIVERSITY OF OREGON

UNIVERSITY OF SOUTHERN CALIFORNIA

STANFORD UNIVERSITY

UNIVERSITY OF HAWAII

UNIVERSITY OF TOKYO

UNIVERSITY OF UTAH

WASHINGTON STATE UNIVERSITY

UNIVERSITY OF WASHINGTON 


\section{Pacific Journal of Mathematics}

Vol. 144, No. $1 \quad$ May, 1990

Wojciech Chojnacki, On some totally ergodic functions $\ldots \ldots \ldots \ldots \ldots \ldots 1$

Steven R. Costenoble, Stefan Waner and G. S. Wells, Approximating

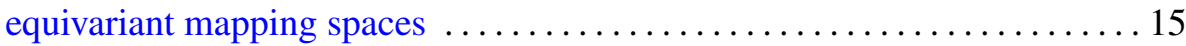

Peter Michael Higgins, A short proof of Isbell's zigzag theorem . . . . . . . 47

Harold H. Johnson, The absolute invariance of conservation laws ........5 51

Edgar Kann, Infinitesimal rigidity of almost-convex oriented polyhedra of arbitrary Euler characteristic $\ldots \ldots \ldots \ldots \ldots \ldots \ldots \ldots \ldots \ldots \ldots \ldots \ldots$

Alan Van Lair, Uniqueness for a nonlinear abstract Cauchy problem . . . . . 105

John B. Little and Kathryn A. Furio, On the distribution of Weierstrass points on irreducible rational nodal curves $\ldots \ldots \ldots \ldots \ldots \ldots \ldots \ldots \ldots 131$

J. S. Okon and Louis Jackson Ratliff, Jr., Reductions of filtrations . . . . . 137

Janusz Pawlikowski, Small subset of the plane which almost contains almost all Borel functions ............................... 155

Sergio A. Tozoni, Vector singular integral operators on a local field ....... 161 John Bason Wagoner, Triangle identities and symmetries of a subshift of

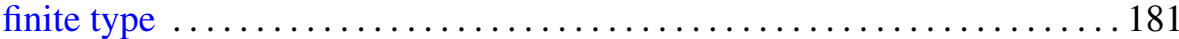

